\title{
An Easy Calibration Method for Central Catadioptric Cameras
}

\begin{abstract}
DENG Xiao-Ming $^{1} \quad$ WU Fu-Chao $^{1} \quad$ WU Yi-Hong ${ }^{1}$
Abstract Central catadioptric cameras are widely used in virtual reality and robot navigation, and the camera calibration is a prerequisite for these applications. In this paper, we propose an easy calibration method for central catadioptric cameras with a $2 \mathrm{D}$ calibration pattern. Firstly, the bounding ellipse of the catadioptric image and field of view (FOV) are used to obtain the initial estimation of the intrinsic parameters. Then, the explicit relationship between the central catadioptric and the pinhole model is used to initialize the extrinsic parameters. Finally, the intrinsic and extrinsic parameters are refined by nonlinear optimization. The proposed method does not need any fitting of partial visible conic, and the projected images of $2 \mathrm{D}$ calibration pattern can easily cover the whole image, so our method is easy and robust. Experiments with simulated data as well as real images show the satisfactory performance of our proposed calibration method.
\end{abstract}

Key words Central catadioptric camera, calibration, 2D calibration pattern, computer vision

\section{Introduction}

Catadioptric cameras have wide field of view (FOV) and they are widely used in many applications such as virtual reality, robot navigation, and visual surveillance. The calibration of catadioptric cameras is a prerequisite for these applications. Central catadioptric cameras, a kind of catadioptric cameras that have a unique viewpoint, have attracted increasing attentions in recent years ${ }^{[1]}$. This paper provides an easy calibration method for the central catadioptric cameras.

The previous calibration methods for the central catadioptric cameras could be classified into the following five categories:

1) Self-calibration without scene information. Kang ${ }^{[2]}$ used the consistency of pair-wise tracked point features for calibration. The method is only suitable for catadioptric cameras with a paraboloidal mirror.

2) Sphere based calibration. Ying and $\mathrm{Hu}^{[3]}$ analyzed the relation of the camera intrinsic parameters and the sphere imaged contour. Then, they applied the relation to calibrating central catadioptric cameras.

3) Line based calibration. Geyer and Daniilidis ${ }^{[4]}$ calibrated para-catadioptric cameras from at least three line images. Barreto and Araujo ${ }^{[5]}$ studied the geometric properties of line images under the central catadioptric model, and gave a calibration method for all kinds of central catadioptric cameras. All these methods involve conic fitting, an error-prone process when only partial contour is available. Recently, Wu et al. ${ }^{[6]}$ introduced a shift from the central catadioptric model to the pinhole model, then a constraint that after the above shift the image points on a space line must be collinear was used to calibrate paracatadioptric-like cameras. This method did not need fittings of partially visible conic and was totally linear.

4) Point based calibration. From known 3D space points, Aliaga $^{[7]}$ proposed an approach to estimate camera intrinsic and extrinsic parameters, where the mirror center was manually determined. Vasseur and Mouaddib ${ }^{[8]}$ calculated intrinsic parameters by a nonlinear method with 3D space points. $\mathrm{Wu}$ and $\mathrm{Hu}^{[9]}$ introduced the invariants of

Received August 21, 2006; in revised form April 17, 2007

Supported by National Natural Science Foundation of China (60575019), the National High Technology Research and Development Program of China (863 Program) (2006AA01Z116), and Institute of Automation Chinese Academy of Sciences Innovation Fund For Young Scientists

1. National Laboratory of Pattern Recognition, Institute of Automation, Chinese Academy of Sciences, Beijing 100080, P. R. China DOI: $10.1360 /$ aas-007-0801
$1 \mathrm{D} / 2 \mathrm{D} / 3 \mathrm{D}$ space points and then used them to compute the camera principal point with a quasi-linear method.

5) 2D calibration method. Recently, Kannata and Brandt ${ }^{[10]}$ proposed a $2 \mathrm{D}$ calibration method for fisheye cameras, and Scaramuzza et al. ${ }^{[11]}$ proposed a $2 \mathrm{D}$ calibration method for catadioptric cameras. Since the images of 2D pattern can easily cover the whole catadioptric image, their methods are capable of accurate calibration. However, the single view point constraint, which is of great importance to the central catadioptric cameras ${ }^{[1]}$, was not used in the two papers.

This work is inspired by Kang ${ }^{[2]}$. Kang proposed a direct circle-based self-calibration method, which used the center of the extracted bounding ellipse of the omnidirectional image as the principal point and got the mirror parameter with the FOV and radius of the omnidirectional image. Kang's method can be used only for catadioptric cameras with a paraboloidal mirror. In Kang's paper, the conclusion is that the circle-based method can on occasion produce reasonable results.

In this paper, we propose an easy calibration method for central catadioptric cameras with a $2 \mathrm{D}$ calibration pattern. Our method is suitable for central catadioptric cameras with all kinds of quadric mirrors (including planar mirrors), whose FOVs are smaller than or equal to full hemisphere i.e. FOVs $\leq 180^{\circ}$. With the bounding ellipse of the catadioptric image and the camera's FOV, the initial intrinsic parameters can be obtained under the unified image model $^{[12]}$. Next, we take one or more images of the $2 \mathrm{D}$ calibration pattern under different orientations to refine the intrinsic parameters ${ }^{1}$. Although the explicit relationship between the central catadioptric and the pinhole model is not new ${ }^{[6]}$, in this paper, we give its geometric interpretation and use the above explicit relationship to initialize the extrinsic parameters of $2 \mathrm{D}$ calibration pattern. Optimization of the reprojection error is finally engaged to refine all the parameters. Experiments show that our method can always get good calibration results with both simulated and real image data.

The advantages of our paper lie in the following aspects:

1) No need for fitting partial visible conic. It is a hard problem $^{[13]}$ that most of classical line-based calibrations for a central catadioptric camera need fittings of partial visible conics.

2) Only the bounding ellipse of the catadioptric image

${ }^{1}$ In practice, the catadioptric cameras with $\mathrm{FOV}>180^{\circ}$ can also be calibrated with our method, if the projections of $2 \mathrm{D}$ calibration pattern are all within the region projected via the below hemisphere. 
and several images of $2 \mathrm{D}$ calibration pattern are needed for a full calibration. With more images of a $2 \mathrm{D}$ calibration pattern, the accuracy and robustness of calibration can be further increased.

3) It is an easy calibration method for both pinhole and central catadioptric cameras with all kinds of quadric mirrors.

This paper is organized as follows. In Section 2, the pinhole and central catadioptric camera models are introduced. Section 3 elaborates on how to calibrate central catadioptric camera with points on a plane. All the intrinsic and extrinsic parameters can be analytically obtained. Section 4 reports some experiments with both simulated and real image data. Section 5 is the conclusion.

\section{Preliminaries}

\subsection{Pinhole camera model}

Under the pinhole camera model, a $3 \mathrm{D}$ point $\boldsymbol{M}$ is projected to its image point $\boldsymbol{m}$ by

$$
\boldsymbol{m} \approx K[R, \boldsymbol{t}]\left[\begin{array}{c}
\boldsymbol{M} \\
1
\end{array}\right], K=\left[\begin{array}{ccc}
r f & s & u_{0} \\
0 & f & v_{0} \\
0 & 0 & 1
\end{array}\right]
$$

where $R$ is the rotation matrix and $t$ is the translation vector, $K$ is the camera intrinsic matrix, with $f$ the focal length, $r$ the aspect ratio, $\left[u_{0}, v_{0}\right]$ the principal point, and $s$ the parameter describing the skew of the two image axes.

\subsection{Central catadioptric camera model}

Catadioptric cameras are devices of pinhole cameras mounted with a mirror. Baker and Nayar ${ }^{[1]}$ investigated that the revolving mirrors for catadioptric cameras with a single viewpoint are planar, paraboloidal, hyperbolical, and ellipsoidal. A catadioptric camera consists of reflective (catoptric) and refractive (dioptric) steps (as shown in Fig. 1): an incident ray from a $3 \mathrm{D}$ point $\boldsymbol{M}$ is reflected by a quadric mirror, then the reflected ray is refracted through the pinhole camera onto the image plane.

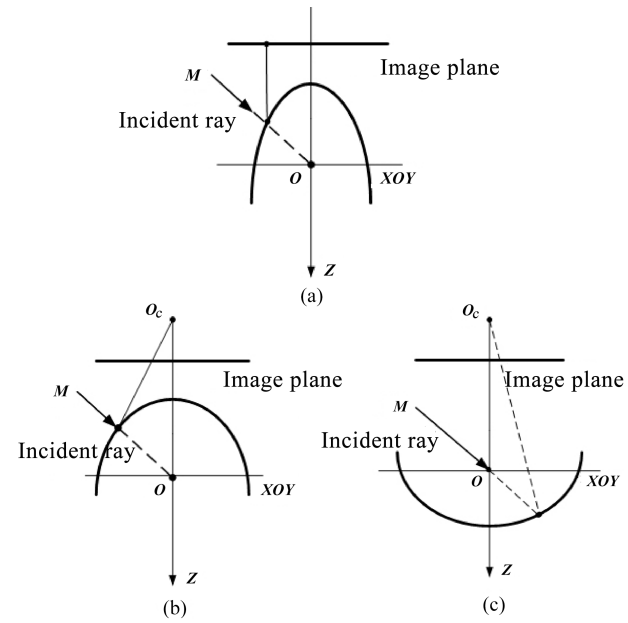

Fig. 1 Central catadioptric cameras with quadric mirrors (where $\boldsymbol{O}$ is the single viewpoint and $\boldsymbol{Z}$ axis is the optical axis) ((a) Paraboloidal mirror; (b) Ellipsoidal mirror; (c) Hyperbolical mirror. For central catadioptric cameras with ellipsoidal and hyperbolical mirrors, the optical center of pinhole camera and the viewpoint $\boldsymbol{O}$ are at the two foci of ellipsoid and hyperboloid. For a central catadioptric camera with paraboloidal mirror, the incident ray from $M$ is reflected by the mirror, then the reflected ray is orthographically projected to the image plane.)
Geyer and Daniilidis ${ }^{[12]}$ proposed a generalized image formation model for central catadioptric cameras. They showed that the above central catadioptric image formation is equivalent to the following two-step mapping by a sphere (see Fig. 2).

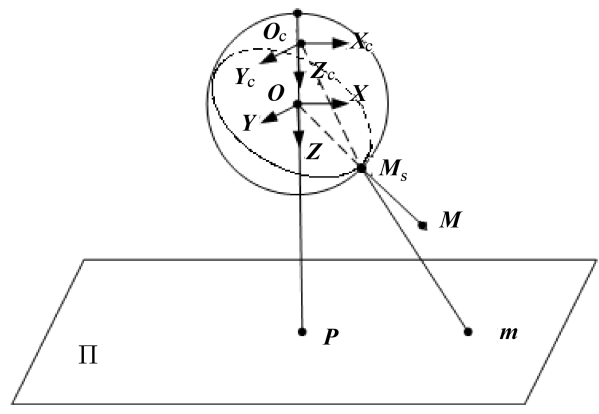

Fig. 2 Image formation of a central catadioptric camera

A 3D point $\boldsymbol{M}$ is projected to a point $\boldsymbol{M}_{s}$ on a unit sphere centered at the viewpoint $\boldsymbol{O}$, then projected to a point $\boldsymbol{m}$ on the image plane $\Pi$ by a virtual pinhole camera through the perspective center $\boldsymbol{O}_{c}$. The image plane is perpendicular to the line of the viewpoints $\boldsymbol{O}$ and $\boldsymbol{O}_{c}$, and it is called the catadioptric image plane. The process can be explicitly expressed as ${ }^{[6]}$

$$
\boldsymbol{m} \approx K\left(\frac{R \boldsymbol{M}+\boldsymbol{t}}{\|R \boldsymbol{M}+\boldsymbol{t}\|}+[0,0, \xi]^{\mathrm{T}}\right)
$$

The optical axis of the pinhole camera is the line $\boldsymbol{O} \boldsymbol{O}_{c}$ and thus its principal point $\boldsymbol{p}=\left[u_{0}, v_{0}, 1\right]^{\mathrm{T}}$ is the intersecting point of the line $\boldsymbol{O O}_{c}$ with the image plane $\Pi$. The distance from point $\boldsymbol{O}$ to $\boldsymbol{O}_{c}, \xi=\left\|\boldsymbol{O}-\boldsymbol{O}_{c}\right\|$, which is commonly called the mirror parameter, determines the mirror used in the central catadioptric camera. The mirror is a paraboloid if $\xi=1$, an ellipsoid or hyperboloid if $0<\xi<1$, and a plane if $\xi=0$.

\section{Calibration algorithm}

In our method, the mirror parameter $\xi$, FOV of the catadioptric camera is assumed to be known, and the bounding ellipse of the catadioptric image is supposed to be visible.

\subsection{Calibration of principal point and initializa- tion of skew and aspect ratio}

Before introducing our calibration method, we would like to say that the center of the bounding ellipse of the catadioptric image can be considered as the principal point like in $[3,6]$. The bounding ellipse is fitted with the method described in [13]. If the equation of the fitted boundary ellipse is $a u^{2}+2 b u v+c v^{2}+2 d u+2 e v+f=0$, we can get the principal point as

$$
u_{0}=\frac{b e-c d}{a c-b^{2}}, \quad v_{0}=\frac{b d-a e}{a c-b^{2}}
$$

For the skew and aspect ratio, we use their prior knowledge as the initial values. In practice, this knowledge is known for most cameras. For example, the skew and aspect ratio of many off-the-shelf cameras are close to 0 and 1 , respectively. 


\subsection{Calibration of the focal length}

Let $\boldsymbol{m}=[u, v, 1]^{\mathrm{T}}$ be the projection of the $3 \mathrm{D}$ point $\boldsymbol{M}_{s}=\left[x_{s}, y_{s}, z_{s}\right]^{\mathrm{T}}$ on the unit sphere, and $\boldsymbol{L}=[0,0, \xi]^{\mathrm{T}}$. Then, from (2), we have

$$
\begin{gathered}
\lambda \boldsymbol{m}=K\left[\begin{array}{cccc}
1 & 0 & 0 & 0 \\
0 & 1 & 0 & 0 \\
0 & 0 & 1 & \xi
\end{array}\right]\left[\begin{array}{c}
\boldsymbol{M}_{s} \\
1
\end{array}\right]=K\left(\boldsymbol{M}_{s}+\boldsymbol{L}\right) \\
\boldsymbol{M}_{s}=\lambda K^{-1} \boldsymbol{m}-\boldsymbol{L}
\end{gathered}
$$

Since $\boldsymbol{M}_{s}^{\mathrm{T}} \boldsymbol{M}_{s}=1$, we have

$$
\boldsymbol{m}^{\mathrm{T}} K^{-\mathrm{T}} K^{-1} \boldsymbol{m} \lambda^{2}-2 \boldsymbol{m}^{\mathrm{T}} K^{-\mathrm{T}} \boldsymbol{L} \lambda+\boldsymbol{L}^{\mathrm{T}} \boldsymbol{L}-1=0
$$

Like in [6], two solutions of $\lambda$ from (6) can be obtained as

$$
\lambda_{1,2}=\frac{\boldsymbol{m}^{\mathrm{T}} K^{-\mathrm{T}} \boldsymbol{L} \pm \sqrt{\left(\boldsymbol{m}^{\mathrm{T}} K^{-\mathrm{T}} \boldsymbol{L}\right)^{2}-\boldsymbol{m}^{\mathrm{T}} K^{-\mathrm{T}} K^{-1} \boldsymbol{m}\left(\xi^{2}-1\right)}}{\boldsymbol{m}^{\mathrm{T}} K^{-\mathrm{T}} K^{-1} \boldsymbol{m}}
$$

Since $z_{s}>-\xi$ i.e., $\lambda>0$, we have

$$
\lambda=\frac{\boldsymbol{m}^{\mathrm{T}} K^{-\mathrm{T}} \boldsymbol{L}+\sqrt{\left(\boldsymbol{m}^{\mathrm{T}} K^{-\mathrm{T}} \boldsymbol{L}\right)^{2}-\boldsymbol{m}^{\mathrm{T}} K^{-\mathrm{T}} K^{-1} \boldsymbol{m}\left(\xi^{2}-1\right)}}{\boldsymbol{m}^{\mathrm{T}} K^{-\mathrm{T}} K^{-1} \boldsymbol{m}}
$$

Let $\phi$ be a half of FOV. As shown in Fig. 3, $\phi$ satisfies

$$
\cos \phi=\boldsymbol{M}_{s} \cdot[0,0,1]^{\mathrm{T}}
$$

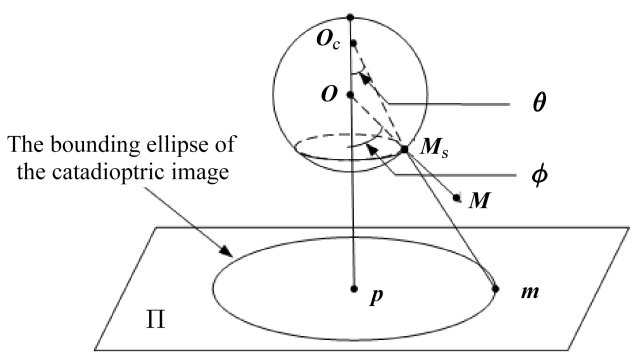

Fig. 3 The FOV of a central catadioptric camera

With the FOV provided by the manufacturer, we can get $\phi=\mathrm{FOV} / 2$. Putting (5) and (8) into (9) and letting $\eta=\boldsymbol{m}^{\mathrm{T}} K^{-\mathrm{T}} K^{-1} \boldsymbol{m}$, we can get

$$
\eta=\frac{2 \xi \cos \phi+\xi^{2}+1}{(\cos \phi+\xi)^{2}}
$$

in which only the focal length $f$ is unknown $^{2}$. Since the skew and aspect ratio are initialized as 0 and 1 , we can get the focal length $f$ using one point $(u, v)$ on the bounding ellipse as

$$
f^{2}=\frac{\left(u-u_{0}\right)^{2}+\left(v-v_{0}\right)^{2}}{\eta-1}
$$

which is a quadratic equation and has two solutions. Since the image plane is always in front of the perspective center $\boldsymbol{O}_{c}$, the positive solution of (11) is chosen as the focal length i.e., $f=\sqrt{\frac{\left(u-u_{0}\right)^{2}+\left(v-v_{0}\right)^{2}}{\eta-1}}$. In addition, the focal length can also be estimated in a geometrical way as shown in the Appendix.

\footnotetext{
${ }^{2} \eta \geq 1$, for $\forall \phi ; \eta=1$, if and only if $\phi=0$.
}

Because the FOV provided by the manufacturer is only an approximation to the true value, there is noise for the selected points on the bounding ellipse, and there also may exist some small misalignments between the mirror and pinhole camera, the above estimated intrinsic parameters can be only approximations. In Sections 3.3 and 3.4, we will use several images of $2 \mathrm{D}$ calibration pattern to refine these initial estimations.

\subsection{Initialization of the extrinsic parameters}

In this part, we use the shift from the central catadioptric model to the pinhole model given in Section III in [6], and then give its geometric interpretation and initialize the extrinsic parameters of $2 \mathrm{D}$ calibration pattern with the homography from the calibration pattern plane to a virtual image plane.

Without loss of generality, we assume the calibration pattern plane is at $\boldsymbol{Z}=0$ of the world coordinate system, thus a point on the calibration pattern plane can be expressed as $\boldsymbol{M}=[x, y, 0]^{\mathrm{T}}$. The extrinsic parameters $(R, \boldsymbol{t})$ can be initialized via the homography $H$ from the calibration pattern plane to a virtual image plane.

Now, we are to estimate $H$. Since

$$
\lambda \boldsymbol{m}=K\left(\frac{R \boldsymbol{M}+\boldsymbol{t}}{\|R \boldsymbol{M}+\boldsymbol{t}\|}+\boldsymbol{L}\right)
$$

we have

$\lambda \boldsymbol{m}-\xi \boldsymbol{p}=K \frac{R \boldsymbol{M}+\boldsymbol{t}}{\|R \boldsymbol{M}+\boldsymbol{t}\|} \approx K(R \boldsymbol{M}+\boldsymbol{t})=K\left[\boldsymbol{r}_{1}, \boldsymbol{r}_{2}, \boldsymbol{t}\right]\left[\begin{array}{l}x \\ y \\ 1\end{array}\right]$

Define $\tilde{\boldsymbol{m}}=\lambda \boldsymbol{m}-\boldsymbol{\xi} \boldsymbol{p}$ with $\lambda$ known as shown in (8) and $\tilde{\boldsymbol{M}}=[x, y, 1]^{\mathrm{T}}$. Then, (13) can be expressed as

$$
\tilde{\boldsymbol{m}} \approx H \tilde{\boldsymbol{M}}
$$

where $H=K\left[\boldsymbol{r}_{1}, \boldsymbol{r}_{2}, \boldsymbol{t}\right]$.

(14) indicates there exists a homography between $\tilde{\boldsymbol{m}}$ and $\tilde{\boldsymbol{M}}$. We should note that the mapping between $\tilde{\boldsymbol{M}}$ and $\boldsymbol{m}$ cannot be described by a homography. This is because, at first, the spacial point is projected on a sphere, not on a plane, then the projected point on the sphere is reprojected on the image plane. Although this whole projection process is from a space plane to the image plane, since the intermediate projection is via a sphere, not a plane, the whole process cannot be modeled as a projective one.

Given the homography and intrinsic matrix, we can obtain $(R, t)$ by

$\boldsymbol{r}_{1}=\mu K^{-1} \boldsymbol{h}_{1}, \boldsymbol{r}_{2}=\mu K^{-1} \boldsymbol{h}_{2}, \boldsymbol{r}_{3}=\boldsymbol{r}_{1} \times \boldsymbol{r}_{2}, \boldsymbol{t}=\mu K^{-1} \boldsymbol{h}_{3}$

where $\mu$ has two solutions, i.e.,

$$
\mu_{1}=\frac{1}{\left\|K^{-1} \boldsymbol{h}_{\mathbf{1}}\right\|}=\frac{1}{\left\|K^{-1} \boldsymbol{h}_{2}\right\|}, \mu_{2}=-\frac{1}{\left\|K^{-1} \boldsymbol{h}_{\mathbf{1}}\right\|}=-\frac{1}{\left\|K^{-1} \boldsymbol{h}_{2}\right\|}
$$

Here, the projections of $2 \mathrm{D}$ calibration pattern are all within the region projected via the below hemisphere, then the projected point $\boldsymbol{M}_{s}$ on a unit sphere, as shown in Fig. 2, is in front of the center of the unit sphere, so we could choose $\mu$

$$
\mu=\operatorname{sign}\left(H_{3,3}\right) \frac{1}{\left\|K^{-1} \boldsymbol{h}_{\mathbf{1}}\right\|}=\operatorname{sign}\left(H_{3,3}\right) \frac{1}{\left\|K^{-1} \boldsymbol{h}_{2}\right\|}
$$

In general, the estimated $R$ is not orthogonal due to noise in data. Therefore, a closest rotation matrix can be 
estimated from $R$ in the sense of the smallest Frobenius norm $^{[14]}$, which can be used to initialize the rotation matrix.

With (13), a geometric interpretation of (14) can be explained as in Fig. 4 (see next page). $\boldsymbol{M}_{s}$ is the projection of a spacial point $\boldsymbol{M}$ (on the calibration pattern) onto the unit sphere. $\boldsymbol{m}$ on $\Pi$ is the projection of the point $\boldsymbol{M}_{s}$ from $\boldsymbol{O}_{c}$, and $\tilde{\boldsymbol{m}}$ on $\tilde{\Pi}$ is the projection of the point $\boldsymbol{M}_{s}$ from $\boldsymbol{O}$. $\Pi$ is the catadioptric image plane, $\tilde{\Pi}$ (parallel to $\Pi$ ) is a virtual image plane, and the principal points of the two image planes $\boldsymbol{p}$ and $\boldsymbol{q}$ are both on the optical axis $\boldsymbol{O} \boldsymbol{O}_{c}$. This is because, as shown in Fig. 2, the image plane $\Pi$ is parallel to the plane $\boldsymbol{O}_{c}-\boldsymbol{X}_{c} \boldsymbol{Y}_{c}$ of the perspective coordinate system $\boldsymbol{O}_{c}-\boldsymbol{X}_{c} \boldsymbol{Y}_{c} \boldsymbol{Z}_{c}$, and the plane $\boldsymbol{O}-\boldsymbol{X} \boldsymbol{Y}$ of the sphere center coordinate system $\boldsymbol{O}-\boldsymbol{X} \boldsymbol{Y} \boldsymbol{Z}$ is parallel to the plane $\boldsymbol{O}_{c}-\boldsymbol{X}_{c} \boldsymbol{Y}_{c}$. From (13), we know that the virtual image plane for $\tilde{\boldsymbol{m}}$ is also parallel to the plane $\boldsymbol{O}-\boldsymbol{X} \boldsymbol{Y}$, so the real and virtual image plane are parallel. Moreover, since the distance between $\boldsymbol{O}$ and $\tilde{\Pi}$ is the focal length, equal to the distance between $\boldsymbol{O}_{c}$ and $\Pi$, the distance between the two parallel planes is $d=\left\|\boldsymbol{O}-\boldsymbol{O}_{c}\right\|=\xi$.

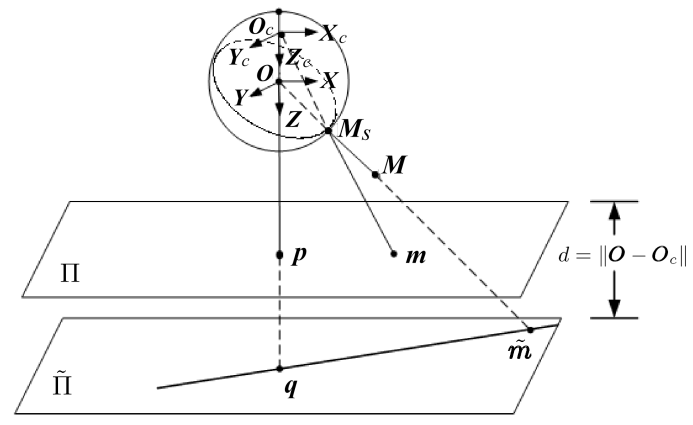

Fig. 4 The geometric interpretation of equation (14)

\section{Remarks}

1) With the calibrated intrinsic parameters, we can always warp a catadioptric image to a perspective one through the sphere center $\boldsymbol{O}$ with an arbitrarily chosen image plane, the chosen virtual plane in this work and its parallel planes in front of the sphere center $\boldsymbol{O}$ could rectify all the view from the below hemisphere, but other perspective planes can only rectify part of the view.

2) If $\xi=0$, i.e., the reflection mirror is a plane mirror, (13) will be changed into

$$
\tilde{\boldsymbol{m}} \approx K(R \boldsymbol{M}+\boldsymbol{t})=K\left[\boldsymbol{r}_{1}, \boldsymbol{r}_{2}, \boldsymbol{t}\right] \tilde{\boldsymbol{M}}
$$

With at least three images of the calibration pattern under different orientations by moving either the camera or pattern, a fully calibration can be done ${ }^{[13]}$ without knowing the FOV or the principal point.

\subsection{Nonlinear optimization}

Let $n$ be the image number of the $2 \mathrm{D}$ calibration plane and $m_{i}$ be the image point number in the $i$-th image of the calibration plane. The calibration is finally optimized by minimizing the following cost function

$$
\min _{K, R_{i}, \boldsymbol{t}_{i}} \sum_{i=1}^{n} \sum_{j=1}^{m_{i}}\left\|\boldsymbol{m}_{i j}-\hat{\boldsymbol{m}}\left(K, R_{i}, \boldsymbol{t}_{i}, \boldsymbol{M}_{j}\right)\right\|^{2}
$$

where $\hat{\boldsymbol{m}}\left(K, R_{i}, \boldsymbol{t}_{i}, \boldsymbol{M}_{j}\right)$ is the image of the point $\boldsymbol{M}_{j}$ in the $i$ th image generated with the estimated $K, R_{i}, \boldsymbol{t}_{i}$. Minimiz- ing (19) can be solved by Levenberg-Marquardt method. The initial estimation of $K, R_{i}, \boldsymbol{t}_{i}$ are given in the above. The rotations $R_{i}$ are expressed with the Rodrigues formula. Since the initial estimation of the principal point is already accurate enough, it can be fixed during the optimization.

\subsection{Our algorithm's outline}

Given the mirror parameter $\xi$ and the FOV, our calibration algorithm is summarized as follows:

1) Take one or more images of $2 \mathrm{D}$ calibration pattern to cover the most of the catadioptric image.

2) Use the bounding ellipse and FOV to initialize the intrinsic parameters as described in Sections 3.1 and 3.2.

3) Compute the homography between $\tilde{\boldsymbol{m}}_{i j}$ and $\tilde{\boldsymbol{M}}_{j}$ using direct linear transformation (DLT) algorithm with data normalization $^{[15]}$, then get the extrinsic parameters $\left(R_{i}, \mathbf{t}_{i}\right)$ as described in Section 3.3.

4) Refine all the intrinsic and extrinsic parameters except the principal point by optimizing (19) as described in Section 3.4 .

\section{Experiments}

In this section, experiments with both simulated and real data are carried out to test our calibration algorithm.

\subsection{Using simulated data}

The simulated camera has the following intrinsic parameter matrix

$$
K=\left[\begin{array}{ccc}
700 & 0.8 & 700 \\
0 & 710 & 750 \\
0 & 0 & 1
\end{array}\right]
$$

The aspect ratio is 0.9859 . The image resolution is of $1500 \times 1500$ pixels. Two catadioptric camera settings with mirror parameters $\xi=0.966$ and $\xi=1.0$, are considered respectively. The $2 \mathrm{D}$ calibration pattern is a checker pattern containing $5 \times 5=25$ markers. The size of the pattern is $44 \mathrm{~cm} \times 44 \mathrm{~cm}$. We generate four movements of the calibration pattern, and the projections are shown in Fig. 5 (a) with $\xi=0.966$ and Fig. 6 (a) with $\xi=1.0$. In Figs 5 (a) and 6 (a), the biggest solid circles are the points on the image of mirror boundary, and the other four types of markers are the projections of the $2 \mathrm{D}$ calibration pattern under four movements. The ground truths of FOVs for the cameras with $\xi=0.966$ and $\xi=1.0$ are $176.2^{\circ}$ and $180^{\circ}$, respectively.

Since the FOV provided by the manufacturer is often an approximation to the true value, we deliberately use the FOVs as $160^{\circ}$ for both the camera settings to test the robustness of our method. The Gaussian noise with 0 mean and standard deviation varying from 0.0 to 5.0 pixels is added to each of the image points on the bounding ellipse and the projected marker image points. At each noise level, we perform 100 independent trials. The means and the standard deviations of $f, r, s, u_{0}, v_{0}$ are computed and shown in Fig. $5(\xi=0.966)$ and Fig. $6(\xi=1.0)$.

From Figs 5 and 6 , the estimated principal points keep close to the ground truth as noise level increases, so it is reliable to use the center of the bounding ellipse as the principal point. In addition, although the estimated focal lengths before optimization deviate much (about 100 pixels) from their ground truths, those after optimization are very close to the true values, and the estimated aspect ratio and skew factor after optimization are all close to their ground truths.

In general, the central part of a catadioptric image is close to a perspective projection, then the extrinsic param- 


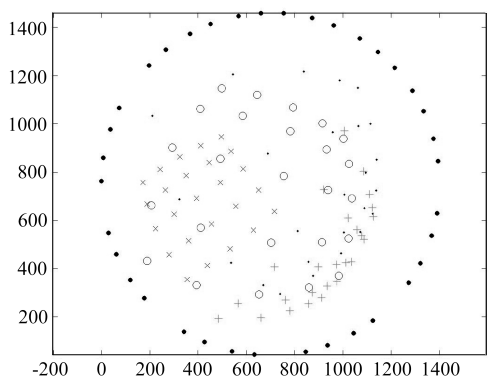

(a)

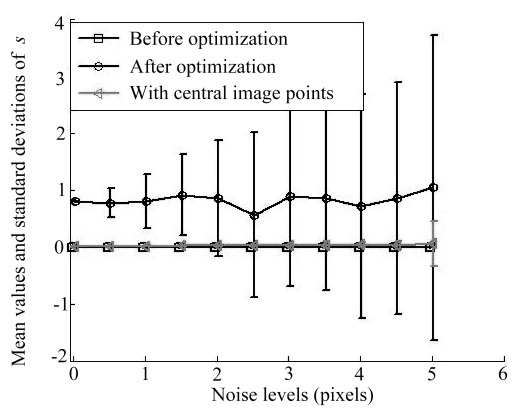

(d)

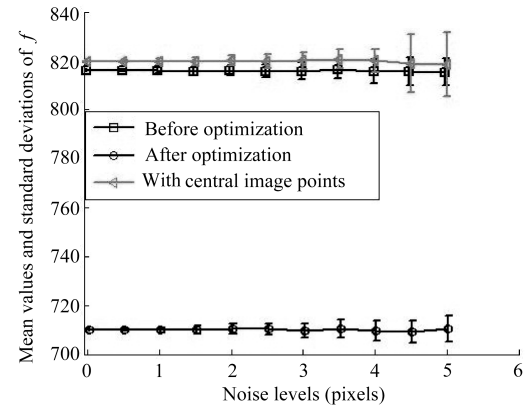

(b)

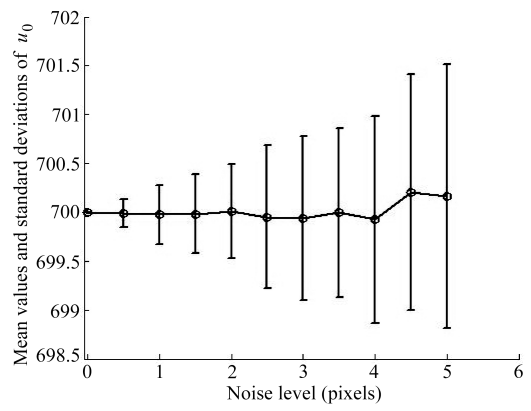

(e)

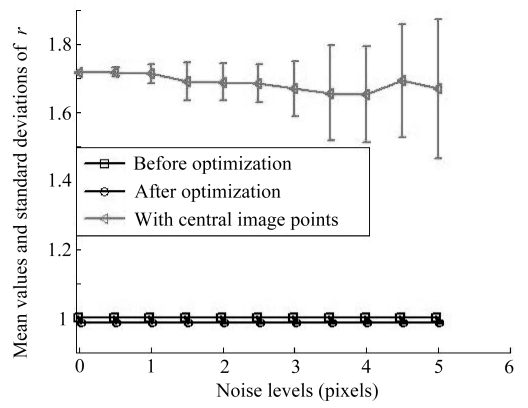

(c)

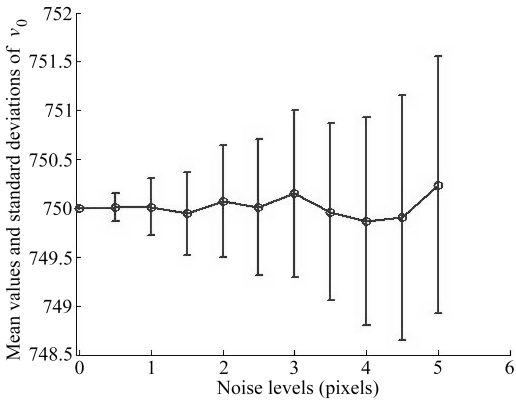

(f)

Fig. 5 Simulated results for a central catadioptric camera with $\xi=0.966$ ((a) The image of the simulated calibration grid (The biggest solid circles are the points on the image of mirror bounding, and the other four types of markers are the projections of $2 \mathrm{D}$ calibration pattern under four movements.); (b) (f) The estimated intrinsic parameters)

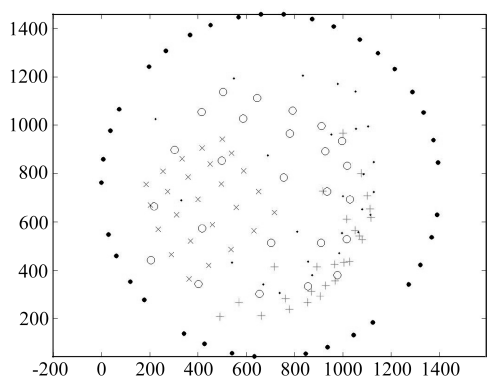

(a)

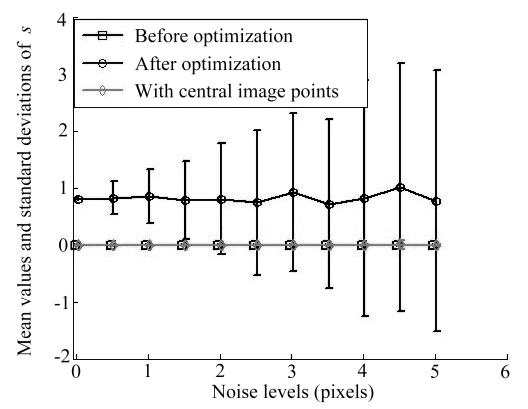

(d)

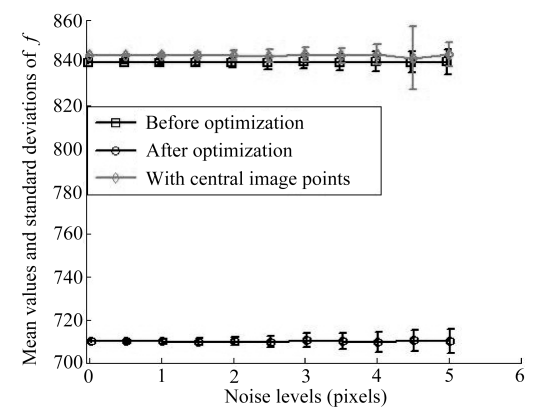

(b)

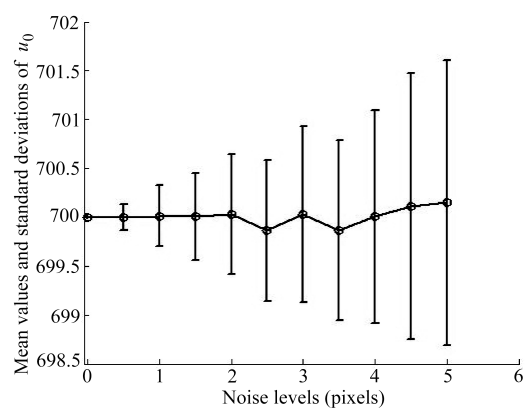

(e)

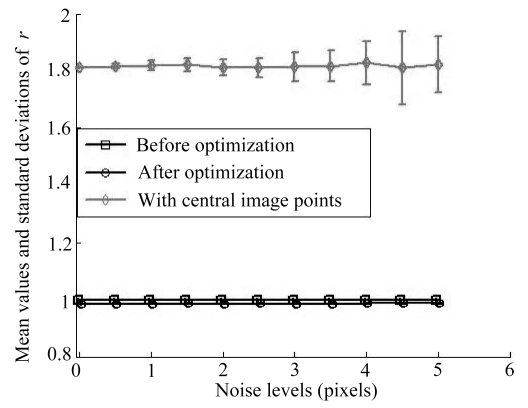

(c)

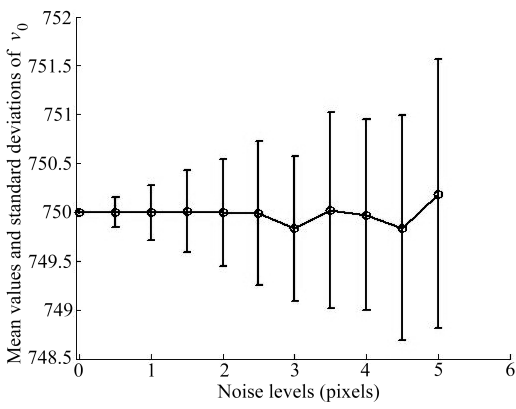

(f)

Fig. 6 Simulated results for a central catadioptric camera with $\xi=1.0$ ((a) The image of the simulated calibration grid (The biggest solid circles are the points on the image of mirror bounding, and the other four types of markers are the projections of $2 \mathrm{D}$ calibration pattern under four movements.); (b) (f) For the estimated intrinsic parameters) 


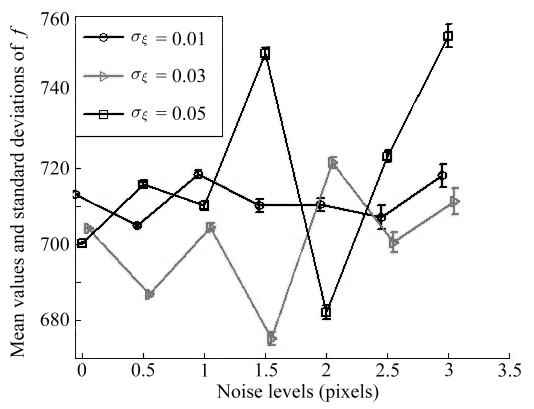

(a)

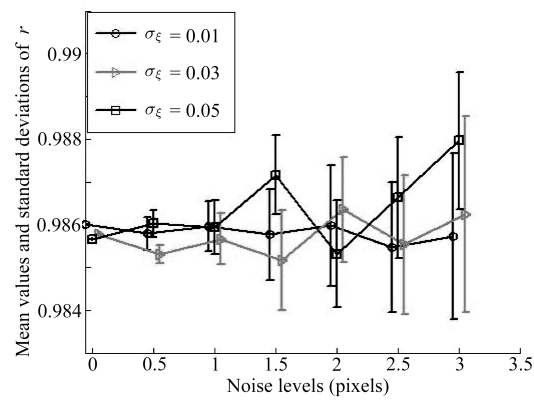

(b)

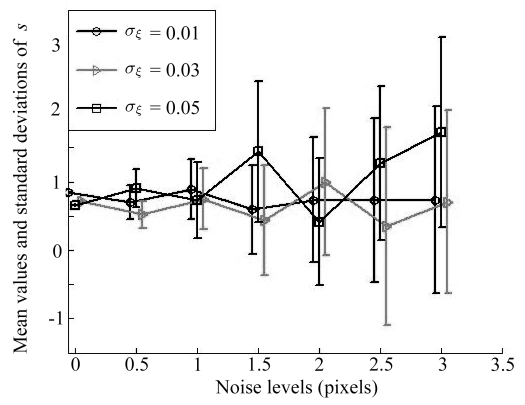

(c)

Fig. 7 The calibrated intrinsic parameters for a central catadioptric camera with $\xi=0.966$, which is contaminated with Gaussian noise with 0 mean and standard deviation $\sigma_{\xi}$ varying from 0.01 to 0.05 . The lines show the intrinsic parameters after optimization.

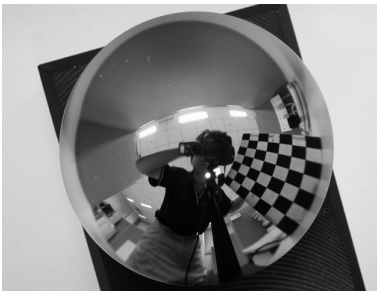

(a)

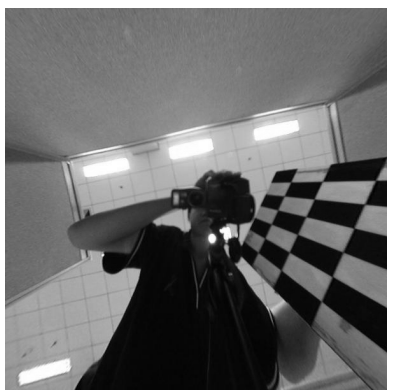

(e)

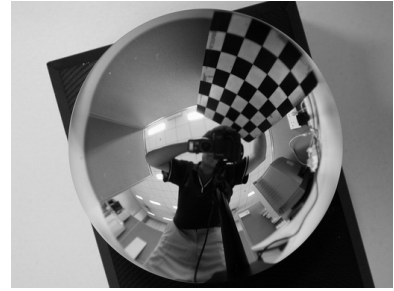

(b)

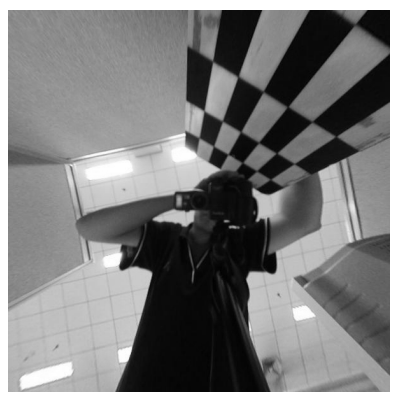

(f)

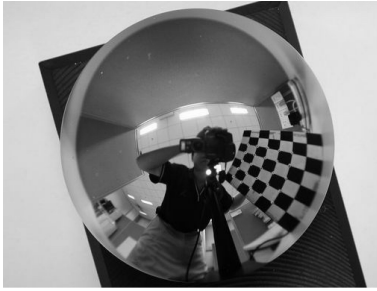

(c)

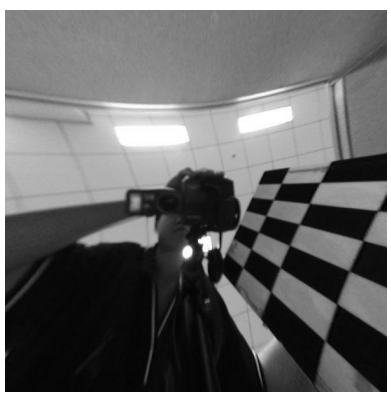

(g)

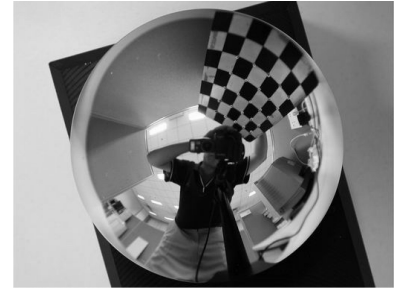

(d)

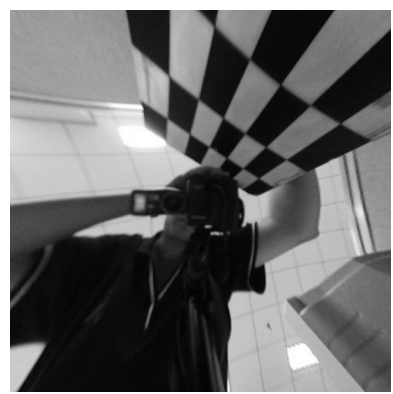

(h)

Fig. 8 Rectifications with calibration results ((a) and (b) Two of the catadioptric images; (c) and (d) The initially chosen and reprojected images of grids with the parameters after optimization; (e) and (f) Rectifications of (a) and (b) with the optimized intrinsic parameters; (g) and (h) Rectifications of (a) and (b) with the line based calibration method in [5])

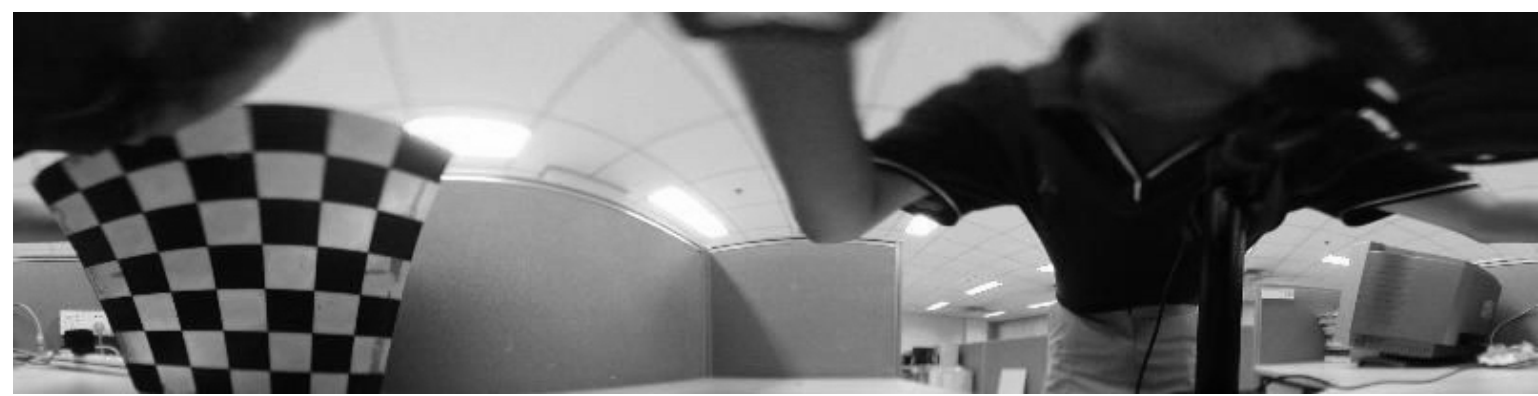

Fig. 9 A panoramic image generated with the calibrated intrinsic parameters of our method 
eters may be also estimated from the homography as [13], which is computed using only central image points. We did make comparisons using the central part of images for estimating the extrinsic parameters. As shown in Figs 5 and 6 , the estimated focal lengths with central part of the catadioptric image after optimization deviate much from the ground truths.

Since the initial focal length is also dependent on the mirror parameter $\xi$, we carry out experiments in which the FOV is deliberately chosen as $160^{\circ}$ and $\xi=0.966$ is contaminated with Gaussian noise. The mean of the noise is equal to 0 , and the standard deviation $\sigma_{\xi}$ varies from 0.01 to 0.05 . Gaussian noise with 0 mean and standard deviation varying from 0.0 to 3.0 pixels are added to each of the image points on the bounding ellipse and the projected marker image points. At each noise level, 100 independent trials are carried out, and the results are shown in Fig. 7. It can be observed that the intrinsic parameters are still close to the ground truths as the noise level increases.

\subsection{Using real image data}

A Nikon CoolPix 990 camera, combined with a hyperboloidal mirror designed by Center of Machine Perception of Czech Technical University, is used. The aspect ratio of the camera is close to 1 and the mirror parameter $\xi$ is 0.966 . The FOV given by the producer is about $217.2^{\circ}$.

Four images of $2 \mathrm{D}$ calibration pattern with the resolution $2048 \times 1536$ pixels were captured during the movements, two of which are shown in Figs 8 (a) and 8 (b). In each of the images, $5 \times 5=25$ projected points are selected manually, and 36 image points on the bounding ellipse of the catadioptric image are chosen by hand. The calibration results before optimization are $f=502.0, r=1.0, s=0.0, u_{0}=$ $1041.9, v_{0}=777.7$, and the results after optimization are $f=528.6, r=1.03, s=1.69, u_{0}=1041.9, v_{0}=777.7$. The originally chosen and reprojected images of Figs 8 (a) and 8 (b) with the optimized parameters almost coincide as shown in Figs 8 (c) and 8 (d). The rectifications of Figs 8 (a) and $8(\mathrm{~b})$ are shown in Figs $8(\mathrm{e})$ and $8(\mathrm{f})$, and the heavily distorted lines are effectively rectified. Fig. 9 is a panoramic image generated with the calibrated intrinsic parameters of our method.

Since there are lines on the 2D calibration pattern, the line based methods can also be used for intrinsic parameter calibration. For each image, the longest line image is selected for calibration with the method in [5]. The calibration results are $f=438.0, r=0.950, s=5.4, u_{0}=1123.1$, and $v_{0}=772.7$. The rectifications of Figs $8(\mathrm{a})$ and $8(\mathrm{~b})$ are shown in Figs $8(\mathrm{~g})$ and $8(\mathrm{~h})$, and the distortions are not effectively removed, which could be because of inaccurate fittings of partial visible conics.

The above simulated and real data experiments both validate the accuracy and robustness of our proposed method.

\section{Conclusion}

In this paper, we propose an easy calibration method for central catadioptric cameras using a $2 \mathrm{D}$ calibration pattern. It can be applied to central catadioptric cameras with all kinds of quadric mirrors. Since the images of the $2 \mathrm{D}$ calibration pattern can easily cover the whole catadioptric image, the robustness is enhanced. In addition, the experiments show that our method is not exigent to the initialization of intrinsic and extrinsic parameters (again thanks to the images of the $2 \mathrm{D}$ calibration pattern). We coin our method "an easy calibration" chiefly because our method needs only capturing one or more images of a $2 \mathrm{D}$ calibration pattern, which is similar to that of Zhang's easy calibration method for a perspective camera with a $2 \mathrm{D}$ pattern $^{[13]}$

\section{References}

1 Baker S, Nayar S. A theory of single-viewpoint catadioptric image formation. International Journal of Computer Vision, 1999, 35(2): 237〜251

2 Kang S B. Catadioptric self-calibration. In: Proceedings of IEEE Conference on Computer Vision and Pattern Recognition. IEEE, 2000. 201 207

3 Ying $\mathrm{X}, \mathrm{Hu} \mathrm{Z}$. Catadioptric camera calibration using geometric invariants. IEEE Transactions on Pattern Analysis and Machine Intelligence, 2004, 26(10): 1260 1271

4 Geyer C, Daniilids K. Paracatadioptric camera calibration. IEEE Transactions on Pattern Analysis and Machine Intelligence, 2002, 24(5): 687 695

5 Barreto J P, Araujo H. Geometry properties of central catadioptric line images and application in calibration. IEEE Transactions on Pattern Analysis and Machine Intelligence, 2005, 27(8): 1327 1333

$6 \mathrm{Wu} \mathrm{Y,} \mathrm{Li} \mathrm{Y,} \mathrm{Hu} \mathrm{Z.} \mathrm{Easy} \mathrm{calibration} \mathrm{for} \mathrm{para-catadioptric-like}$ camera. In: Proceedings of IEEE International Conference on Intelligent Robots and Systems. IEEE, 2006. 5719 5724

7 Aliaga D G. Accurate catadioptric calibration for real-time pose estimation in room-size environments. In: Proceedings of the 8th International Conference on Computer Vision. IEEE, 2001. 127 134

8 Vasseur P, Mouaddib E M. Central catadioptric line detection. In: Proceedings of the British Machine Vision Conference. London, U K: BMVA Press, 2004

$9 \mathrm{Wu} \mathrm{Y,} \mathrm{Hu} \mathrm{Z.} \mathrm{Geometric} \mathrm{invariants} \mathrm{and} \mathrm{applications} \mathrm{under}$ catadioptric camera model. In: Proceedings of International Conference on Computer Vision. IEEE, 2005. 1547 1554

10 Kannata J, Brandt S S. A generic camera model and calibration method for conventenal, wide-eye, and fish-eye lenses. IEEE Transactions on Pattern Analysis and Machine Intelligence, 2006, 28(8): 1335 1340

11 Scaramuzza D, Martinelli A, Siegwart R. A flexible technique for accurate omnidirectional camera calibration and structure from motion. In: Proceedings of International Conference on Computer Vision Systems. IEEE, 2006. 45 45

12 Geyer C, Daniilidis K. Catadioptric projective geometry. International Journal of Computer Vision, 2001, 45(3): $223 \sim 243$

13 Fitzgibbon A, Pilu M, Fisher R B. Direct least square fitting of ellipses. IEEE Transactions on Pattern Analysis and Machine Intelligence, 1999, 21(5): 476 480

14 Zhang Z. A flexible new technique for camera calibration. IEEE Transactions on Pattern Analysis and Machine Intelligence, 2000, 22(11): 1330 1334

15 Hartley R, Zisserman A. Multiple View Geometry in Computer Vision. Cambridge: Cambridge University Press, 2003 


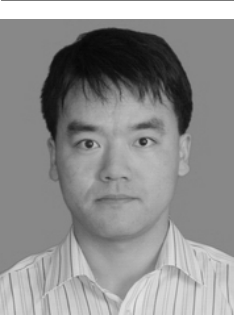

DENG Xiao-Ming Ph. D. candidate at Institute of Automation, Chinese Academy of Sciences. He received his bachelor and master degrees from Wuhan University in 2001 and 2004, respectively. His research interest covers omnidirectional camera calibration and 3D reconstruction. Corresponding author of this paper.

E-mail: xmdeng@nlpr.ia.ac.cn

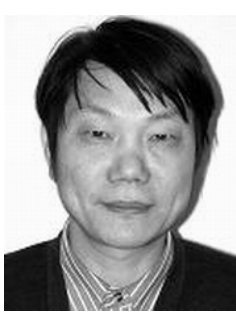

WU Fu-Chao Professor at Institute of Automation, Chinese Academy of Sciences. His research interest covers computer vision, which include camera calibration, 3D reconstruction, active vision, and imagebased modeling and rendering.

E-mail: fcwu@nlpr.ia.ac.cn

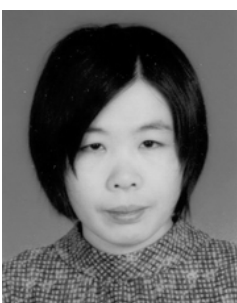

WU Yi-Hong Associate professor at Institute of Automation, Chinese Academy of Sciences. She received her Ph. D. degree from Institute of Systems Science, Chinese Academy of Sciences in 2001. Her research interest covers polynomial elimination and applications, geometric invariant and applications, 3D vision, and object recognition

E-mail:yhwu@nlpr.ia.ac.cn

\section{Appendix: Focal length estimation in a geometrical way}

In Fig. 3, let $\theta=\angle O O_{c} M_{s}$, and it is obvious that $\theta<\frac{\pi}{2}$. We have

$$
f=\left|\boldsymbol{O}_{c} \boldsymbol{p}\right|=\sqrt{\left(u-u_{0}\right)^{2}+\left(v-v_{0}\right)^{2}} \cot \theta
$$

In $\triangle O \boldsymbol{O}_{c} \boldsymbol{M}_{s}$, by using the cosine rule there is

$$
\begin{aligned}
& \left|\boldsymbol{O}_{c} \boldsymbol{M}_{s}\right|=\sqrt{\left|\boldsymbol{O \boldsymbol { O } _ { c }}\right|^{2}+\left|\boldsymbol{O} \boldsymbol{M}_{s}\right|^{2}-2 \cos (\pi-\phi)\left|\boldsymbol{O \boldsymbol { O } _ { c }}\right|\left|\boldsymbol{O} \boldsymbol{M}_{s}\right|} \\
& =\sqrt{\xi^{2}+1+2 \xi \cos \phi}
\end{aligned}
$$

By applying the sine rule to $\triangle O O_{c} M_{s}$, we have

$$
\frac{\sin \theta}{\left|\boldsymbol{O} \boldsymbol{M}_{s}\right|}=\frac{\sin (\pi-\phi)}{\left|\boldsymbol{O}_{c} \boldsymbol{M}_{s}\right|}
$$

Thus

$$
\sin \theta=\sin \phi \frac{\left|\boldsymbol{O} \boldsymbol{M}_{s}\right|}{\left|\boldsymbol{O}_{c} \boldsymbol{M}_{s}\right|}=\frac{\sin \phi}{\sqrt{\xi^{2}+1+2 \xi \cos \phi}}
$$

It follows that

$$
\begin{aligned}
& \cot \theta=\frac{\cos \theta}{\sin \theta}=\frac{\sqrt{1-\sin ^{2} \theta}}{\sin \theta}=\frac{1}{\sqrt{\frac{\xi^{2}+1+2 \xi \cos \phi}{(\xi+\cos \phi)^{2}}-1}}= \\
& \frac{1}{\sqrt{\eta-1}}
\end{aligned}
$$

Therefore,

$$
\eta=\sec ^{2} \theta
$$

Substituting (A3) into (A1), we obtain

$$
f=\sqrt{\frac{\left(u-u_{0}\right)^{2}+\left(v-v_{0}\right)^{2}}{\eta-1}}
$$

(A4) gives a geometrical explanation of $\eta$ and (A5) is the positive solution of (11). 